\title{
Application of Modified Close Value Method in Power Quality Evaluation
}

\author{
Ren Hu${ }^{1, a^{*}}$, Zhaoxia Jing ${ }^{2, b}$, Qinghua Wu ${ }^{3, c}$, Renbao Yan ${ }^{4, d}$, Xin Zhang,e \\ 1,4,5Jiaxing Electric Power Company, State Grid Corporation of China, China \\ ${ }^{1,2,3}$ School of Electric Power, South China University of Technology, China \\ a,d,e 411760670@qq.com, bxjing@scut.edu.cn, cwuqh@scut.edu.cn
}

Keywords: Power quality; Close value method; Analytic hierarchy method; Variation coefficient method; Comprehensive evaluation

\begin{abstract}
For enhancing the accuracy and rationality of power quality (PQ) evaluation, close value method (CVM) based on the target optimization principle is introduced to symbolize the performance of PQ samples. To tackle with the defect, in calculating close value of $P Q$ samples, that the calculation formula of close value is divided by zero, a modified CVM is correspondingly developed to adapt to the randomness of PQ samples. Meanwhile, for analyzing the impacts of weighting approaches on the evaluation outcome, different weighting approaches are orderly utilized to improve CVM. Case analysis shows that the new calculating method of close value is proved to be more simplified and applicable; the impacts different weighting approaches have on the evaluation outcome differ to some extent.
\end{abstract}

\section{Introduction}

Power quality (PQ) is closely related to power supply companies and their economic benefits, as well as the security and reliability of electric power components such as domestic appliances, generators, transformers, etc, which enables PQ evaluation definitely indispensable to formulate the electricity price in accordance with its authentic performance. Presently, PQ evaluation is commonly regarded as a multi-dimension mathematic or multi-attribute decision-making problem that recently researchers have applied numerous evaluation approaches to handle with, such as fuzzy evaluation method, probability theory, projection pursuit method, matter-element analysis, ideal interval method, etc. Most of these methods are on the basis of reduction dimensions, namely, conversion from a multi-dimension problem to a unidimensional problem to compare different PQ samples [1]. In literature [2-5], fuzzy evaluation methods are discussed from the perspective of weighting but the subjective factor of fuzzy subordinating degree functions remains existence. The literature [6] illustrates the utility of probability theory in PQ evaluation which can effectively quantify and normalize the criterion values of PQ, but without considering the criterion weights. Projection pursuit method in $[7,18]$ whose optimal projection vector is vulnerable to various simples is an effective approach to reduce dimensions. Matter-element analysis in [8] is usefully applied to 
the structural establishment and the weighting of criterion, but neglecting the preference information of criterion. Ideal interval method based on the similarity theory is combined with analytic hierarchy method, a subjective weighting approach, which overlooks the significant objective information of PQ samples [19].

Although these approaches above have made extraordinary response to some peculiar problems from diverse perspectives, for mitigating the deficiencies of above methods to some extend, many researchers concentrated on the application of close value method (CVM) in the multi-objective or multi-attribute decision-making of alternatives in the field of aerospace industry, engineering, missile defense system, etc [9-12]. Nevertheless, the situation that the calculating formula of close value of evaluated subject is probably divided by zero with fluctuating samples results in the failure of CVM. Therefore, the modified calculating formula of close value is proposed in this paper and used to conduct PQ evaluation. Additionally, disparate weighting methods are drawn into modifying CVM and comparing their impacts on the evaluation outcome.

\section{Principle of Close Value Method}

There are several procedures to demonstrate the process of CVM. Firstly, a set of PQ samples is collected in the initial decision matrix which is converted into the normalized matrix. Secondly, the set of optimal or pessimal sample from the normalized matrix is catalogued as a benchmark to compute the set of Euclidean distance between each evaluated sample and the optimal or pessimal sample. Finally, calculating close value of each evaluated sample is obtained respectively which implies the priority of all evaluated samples [9-12]. It is assumed that there is a PQ sample set $\boldsymbol{S}=\left[s_{1}, s_{2}, \ldots, s_{m}\right](i=1,2, \ldots, m)$, and a criterion set $\boldsymbol{X}=\left[x_{1}, x_{2}, \ldots, x_{n}\right](j=1,2, \ldots, n)$. Each expression which pertains to CVM can be shown as follows.

\subsection{Normalization of initial decision matrix}

The normalization of initial decision matrix $\mathbf{C}=\left[c_{i j}\right]_{m \times n}$ can be represented by $\mathbf{R}=\left[r_{i j}\right]_{m \times n}$,

$$
r_{i j}= \pm c_{i j} / \sqrt{\sum_{i=1}^{m} c_{i j}^{2}}
$$

where $c_{i j}, r_{i j}(i=1,2, \ldots, m ; j=1,2, \ldots ., n)$ denote the initial value and the normalized value of the criterion $x_{j}$ of PQ sample $s_{i}$. If the criterion $x_{j}$ is a positive one whose value gets larger, the performance of criterion $x_{j}$ will be better, then $r_{i j} \geq 0$; or the criterion $x_{j}$ is a negative one whose value gets smaller, the performance of criterion $x_{j}$ will be better, then $r_{i j}<0$.

\subsection{Definition of the Euclidean distance}

The optimal or pessimal sample can be searched from $\mathbf{R}$ and presented as

$$
\begin{aligned}
& A^{+}=\left\{r_{j}^{+}\right\}=\left\{\max _{1 \leq i \leq m}\left\{r_{i j}\right\}\right\} \\
& A^{-}=\left\{r_{j}^{-}\right\}=\left\{\min _{1 \leq i \leq m}\left\{r_{i j}\right\}\right\}
\end{aligned}
$$

Where $r_{j}{ }^{+}(\mathrm{j}=1,2, \ldots, \mathrm{n})$ is the optimal value of criterion $x_{j}$ within all samples while $r_{j}{ }^{-}(\mathrm{j}=1,2, \ldots$, $\mathrm{n})$ is the pessimal value of criterion $x_{j}$ within all samples. Then the set of Euclidean distance between each evaluated sample and the optimal or pessimal sample can be expressed separately as 


$$
\begin{gathered}
D_{i}^{+}=\left[\sum_{j=1}^{n}\left(w_{j} r_{i j}-w_{j} r_{j}^{+}\right)^{2}\right]^{1 / 2} \\
D_{i}^{-}=\left[\sum_{j=1}^{n}\left(w_{j} r_{i j}-w_{j} r_{j}^{-}\right)^{2}\right]^{1 / 2} \\
\sum_{j=1}^{n} w_{j}=1
\end{gathered}
$$

where $D_{i}{ }^{+}, D_{i}^{-}$successively denote the Euclidean distance between the sample $s_{i}$ and the optimal or pessimal sample. And $w_{j}$ signifies the weight of criterion $x_{j}$.

\subsection{Calculating formula of close value}

The calculating formula of close value is deduced as

$$
E_{i}=\frac{D_{i}^{+}}{\min \left\{D_{i}^{+}\right\}}-\frac{D_{i}^{-}}{\max \left\{D_{i}^{-}\right\}}
$$

Where $E_{i}$ named the close value of sample $s_{i}$ indicates the extent of closeness to the optimal sample and estrangement to the pessimal sample. And the smaller $E_{i}$ gets, the closer to the optimal the sample will be.

\section{Demonstration of Modified Close Value Method}

\subsection{Modified formula of close value}

Considering the fluctuation of PQ samples which prompts the possibility that $\min \left\{D_{i}^{+}\right\}=0$ or $\max \left\{D_{i}^{-}\right\}=0$ exists may encounter with the failure of performing CVM [9-12], a modified formula of close value is correspondingly developed as

$$
E_{i}=\frac{D_{i}^{+}}{D_{i}^{-}}
$$

Note that $E_{i}=0$ means the sample $s_{i}$ is closest to the optimal, otherwise, $E_{i}=\infty$ suggests the sample $s_{i}$ is equivalent to the pessimal.

\subsection{Discussion of different empowerment methods}

For this reason that some literatures applies the CVM provided that all the criteria are empowered with the equal weight [11,12], in this study different weighting approaches such as Analytic hierarchy method (AHP), Variation coefficient method (VCM) and synthetical empowerment (SE), are separately introduced in CVM to discuss the influences on the assessment result.

AHP is a subjective empowerment method based on the structural framework of PQ evaluation [13]. Firstly, the hierarchical structure constituted by target layer, criterion layer and index layer is established. Then, forming pair comparison matrices is followed by the ranking of each layer and the consistency verification. Finally, the overall ranking of index layer is performed to determine the index weight vector $\boldsymbol{U}=\left[u_{1}, u_{2}, \ldots \ldots . u_{n}\right]$.

Reversely, VCM is an objective empowerment method which traces the fluctuation of differentiation information of sample to compute the variable coefficient of index that hints the 
importance or weight of index represented as $\boldsymbol{V}=\left[v_{1}, v_{2}, \ldots . . . v_{\mathrm{n}}\right]$,

$$
v_{i}(r)=\left|\delta_{i} / R_{i}\right| /\left(\sum_{i=1}^{n}\left|\delta_{i} / R_{i}\right|\right)
$$

Where $\delta_{i}, R_{i}, \delta_{i} / R_{i}$ ordinally denote the standard deviation, the average value, and the variable coefficient of index [14].

Then the combination of AHP and VCM formulates SE which can be expressed as $W=\left[w_{1}, w_{2}, \ldots \ldots w_{n}\right]$, indicating the index weight vector [4],

$$
w_{i}=u_{i} v_{i} / \sum_{j=1}^{n} u_{j} v_{j}
$$

\section{Index Framework of Power Quality Evaluation}

The indices of PQ Evaluation can be mainly catalogued into the technical index set which consists of voltage quality indices (such as voltage deviation (VD), voltage sags (VS), voltage flicker (VF1), three-phase unbalance (TPU), voltage fluctuation (VF2), Harmonic distortion (HD)), frequency deviation (FD), power supply reliability (PSR) and the non-technical index like service satisfactory (SS). The index framework of PQ evaluation can be deployed in Figure 1.

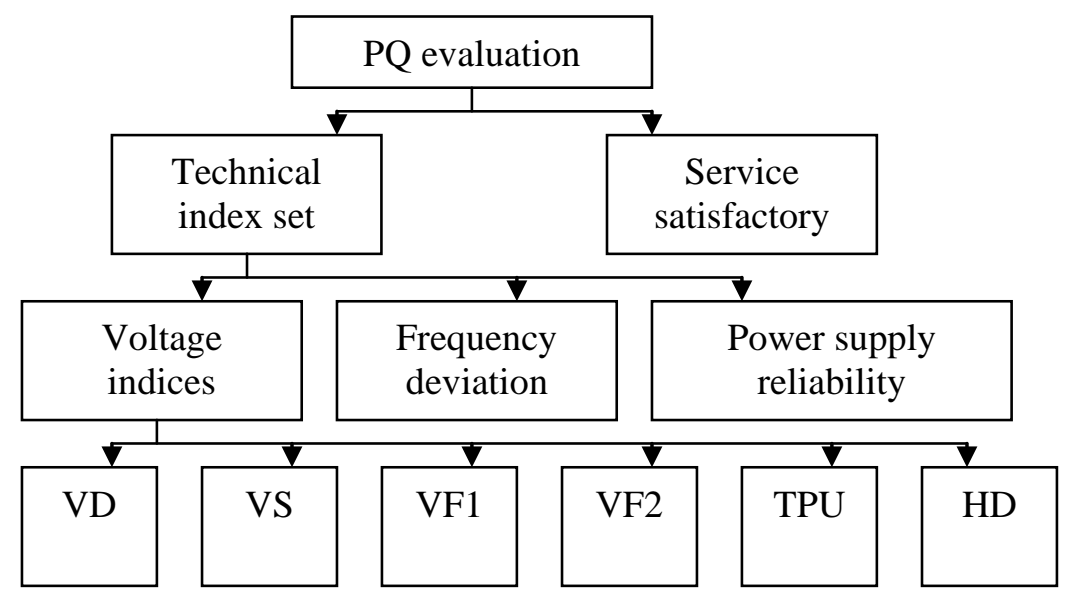

Figure 1 The index framework of PQ evaluation

Recent years have also seen the gradually accumulating achievements relevant to PQ standards of China which define the general PQ index set and can be excerpted as a cardinal limit criterion of PQ. The index grade boundaries of PQ evaluation can be showed in Table 1.

Table 1 Index grade boundaries of PQ evaluation

\begin{tabular}{cccccc}
\hline index/grade NO. & 1 & 2 & 3 & 4 & 5 \\
\hline VD (\%) & $\leqslant 1.20$ & $\leqslant 3.00$ & $\leqslant 4.50$ & $\leqslant 7.00$ & $>7.00$ \\
VS & $\geqslant 0.90$ & $\geqslant 0.80$ & $\geqslant 0.50$ & $\geqslant 0.10$ & $<0.10$ \\
TPU (\%) & $\leqslant 0.50$ & $\leqslant 1.00$ & $\leqslant 1.50$ & $\leqslant 2.00$ & $>2.00$ \\
VF2 (\%) & $\leqslant 0.50$ & $\leqslant 1.00$ & $\leqslant 1.50$ & $\leqslant 2.00$ & $>2.00$ \\
VF1 & $\leqslant 0.20$ & $\leqslant 0.50$ & $\leqslant 0.80$ & $\leqslant 1.00$ & $>1.00$ \\
HD (\%) & $\leqslant 1.00$ & $\leqslant 2.00$ & $\leqslant 3.00$ & $\leqslant 5.00$ & $>5.00$ \\
FD & $\leqslant 0.05$ & $\leqslant 0.10$ & $\leqslant 0.15$ & $\leqslant 0.20$ & $>0.20$ \\
PSR & $\geqslant 0.95$ & $\geqslant 0.85$ & $\geqslant 0.80$ & $\geqslant 0.70$ & $<0.70$ \\
SS & $\geqslant 0.90$ & $\geqslant 0.80$ & $\geqslant 0.70$ & $\geqslant 0.60$ & $<0.60$ \\
\hline
\end{tabular}


In accordance with current national standards, in Table 1 PQ are generally divided into five grades where voltage sags (VS), power supply reliability (PSR), service satisfactory (SS) are compiled as negative index characterising that its smaller value flavors the evaluation result. Reversely, except above negative index set, other indices in Table 1 are expressed as positive index which gets larger, the evaluation result may be better.

\section{Numerical Case analysis}

Analyzing the failure of CVM associated with its incomplete formula of close value is firstly discussed in this study by five random samples of PQ as well as coupled with a substantiation of new formula. Then another case of eight random samples of PQ is demonstrated under the modified $\mathrm{CVM}$ to compare the complicated influences from diverse empowerment approaches on the case result.

\subsection{Assessment of five random samples of $P Q$}

According to [7], five random samples of PQ collected from Shapinba district of Chongqin in China can be cited to assess their performances and obtaining $D^{+} 、 D^{-}$are respectively depicted by equation (1) to (6) as below.

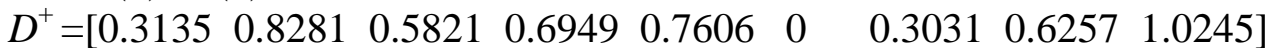

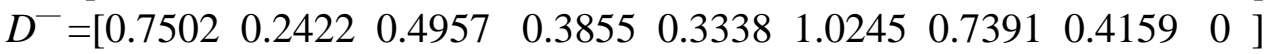

Apparently, $\min \left\{D^{+}\right\}=0$ contributes to the invalidity of CVM and the disruption of evaluation and ranking which manifests the vulnerability of CVM to random samples. Hence, the new formula of close value, equation (8), is applied to compute the case result listed in the Table 2.

Table 2 evaluation result of 5 samples

\begin{tabular}{cccccc}
\hline Sample & $s_{1}$ & $s_{2}$ & $s_{3}$ & $s_{4}$ & $s_{5}$ \\
\hline Ranking & 3 & 4 & 3 & 4 & 4 \\
\hline
\end{tabular}

The final ranking from Table 2 shows the modified CVM can tackle with the situation of unsuccessfully calculating close value and make clear.

\subsection{Assessment of eight random samples of $P Q$}

For the further demonstration of the validity of modified CVM and the impacts of different empowerment approaches on the evaluation, another eight random samples of PQ from [3] are similarly gathered and shown in the initial decision-making matrix $C$

$$
C=\left[\begin{array}{lllllllll}
52.44 & 4.80 & 0.122 & 0.83 & 1.33 & 2.72 & 0.54 & 0.54 & 0.832 \\
75.89 & 1.68 & 0.062 & 0.36 & 0.53 & 1.28 & 0.33 & 0.83 & 0.713 \\
3.56 & 4.35 & 0.180 & 1.35 & 1.95 & 4.67 & 0.93 & 0.32 & 0.864 \\
17.30 & 6.38 & 0.177 & 1.74 & 1.37 & 3.36 & 0.84 & 0.54 & 0.684 \\
62.30 & 3.22 & 0.102 & 0.83 & 0.88 & 2.57 & 0.32 & 0.76 & 0.783 \\
31.30 & 4.69 & 0.138 & 1.14 & 1.67 & 3.31 & 0.66 & 0.67 & 0.765 \\
17.60 & 4.74 & 0.144 & 1.09 & 1.46 & 2.79 & 0.57 & 0.75 & 0.841 \\
19.20 & 6.20 & 0.186 & 1.34 & 1.57 & 4.06 & 0.76 & 0.31 & 0.697 \\
90 & 1.20 & 0.05 & 0.50 & 0.50 & 1.00 & 0.20 & 0.95 & 0.90 \\
80 & 3.00 & 0.10 & 1.00 & 1.00 & 2.00 & 0.50 & 0.85 & 0.80 \\
50 & 4.50 & 0.15 & 1.50 & 1.50 & 3.00 & 0.80 & 0.80 & 0.70 \\
10 & 7.00 & 0.20 & 2.00 & 2.00 & 5.00 & 1.00 & 0.70 & 0.60
\end{array}\right]
$$


Then, $A^{+}$and $A^{-}$ensue the normalized matrix $\mathrm{C}$, which are deduced as

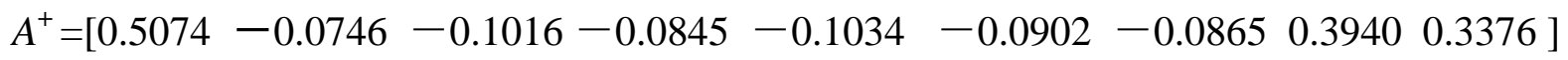

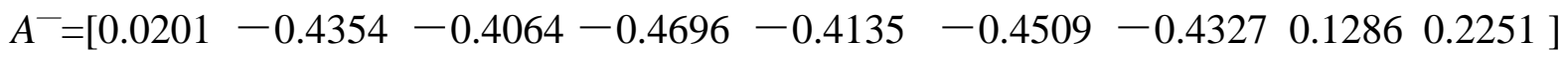

Through AHP, VCM, and SE, Calculated index weight vectors $\boldsymbol{U}, \boldsymbol{V}, \boldsymbol{W}$, can also be listed orderly as below.

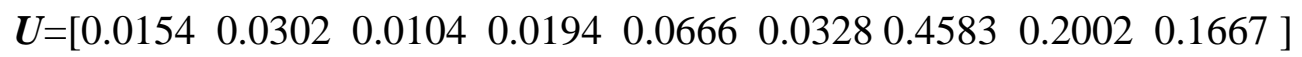

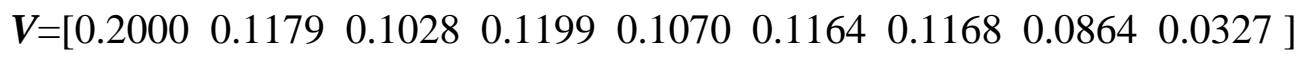

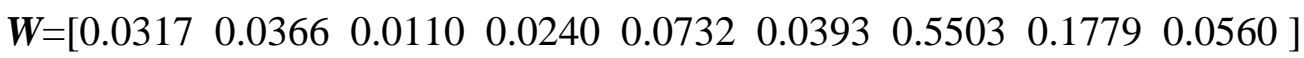

With the application of CVM with equitable weight (CVM1), CVM with AHP (CVM2), CVM with VCM (CVM3), and CVM with SE (CVM4), the corresponding close value set can be separately represented as $E, E_{1}, E_{2}, E_{3}$

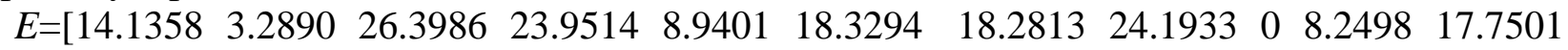
29.3988]

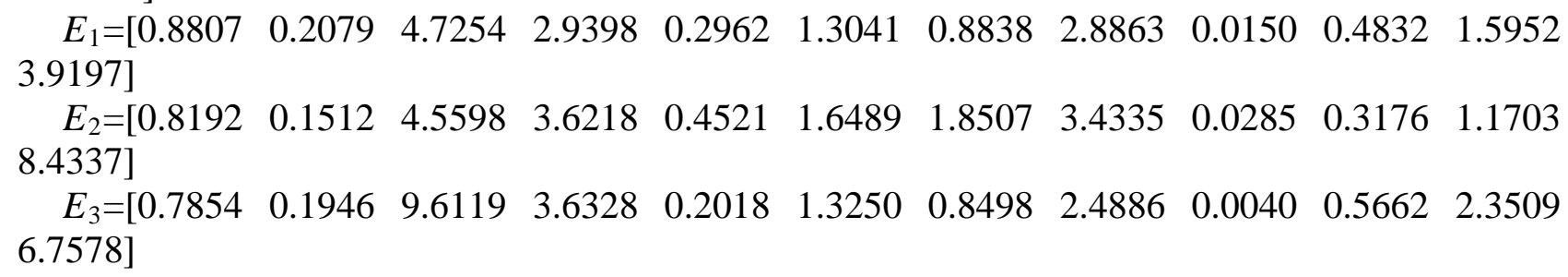

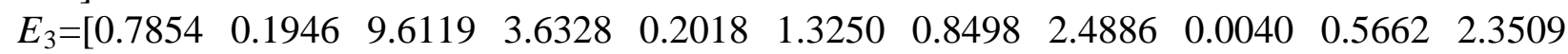
6.7578]

Therefore, the final assessment results derive from CVM1 to CVM4 and [3, 5, 8, 17] can be compared in the Table 3.

Table 3 ranking results of PQ by various methods

\begin{tabular}{ccccccccc}
\hline Sample & $s_{1}$ & $s_{2}$ & $s_{3}$ & $s_{4}$ & $s_{5}$ & $s_{6}$ & $s_{7}$ & $s_{8}$ \\
\hline CVM1 & 3 & 2 & 4 & 4 & 3 & 4 & 4 & 4 \\
CVM2 & 3 & 2 & 4 & 4 & 2 & 3 & 3 & 4 \\
CVM3 & 3 & 2 & 4 & 4 & 3 & 4 & 4 & 4 \\
CVM4 & 3 & 2 & 5 & 4 & 2 & 3 & 3 & 4 \\
{$[3]$} & 1 & 3 & 5 & 4 & 2 & 3 & 3 & 4 \\
{$[17]$} & 2 & 4 & 5 & 4 & 2 & 3 & 2 & 4 \\
{$[5]$} & 3 & 2 & 4 & 4 & 2 & 3 & 3 & 4 \\
{$[8]$} & 2 & 4 & 5 & 4 & 2 & 3 & 3 & 4 \\
\hline
\end{tabular}

Through Table 3 there are some findings as follow:

(1) As for CVM1 CVM4, the result of each method is basically similar. Meanwhile, the ranking of CVM1 is totally the same with CM3's, while the ranking of CVM2 is almost the same with CVM4's. Whereas, the key nuance between CVM with objective empowerments-CVM1, CVM3, and CVM with subjective empowerments-CVM2, CVM4, is attributed to the judgment of $s_{5} \sim s_{7}$ which manifests CVM containing subjective information such as expertise prefers much more optimistic ranking.

(2)The results from CVM1 CVM4 and [5] are almost consistent while those of [3], [8] and [17] are basically similar. Actually, the key discrepancy concentrates on the priority of $s_{1}$ and $s_{2}$. Through the original data of each index from the initial matrix C, the positive index group of $s_{2}$ except for SS are virtually superior to those of $s_{1}$. Similarly, the negative index group of $s_{1}$ are seen 
to be inferior to those of $s_{2}$, though $s_{1}$ holds a higher PSR. Therefore, $s_{2}$ is more likely to be preferential than $s_{1}$ provided each index is configured with apt weight; that's to say, CVM1 CVM4 and [5] may get a more rational assessment in this circumstance.

\section{Conclusion}

The developed new formula of CVM is valid and simplified to answer its invalidity of CVM when random samples evaluated lead to the formula of close value divided by zero. And diverse empowerment approaches introduced in CVM are discussed to demonstrate special influences on the final judgment of samples and conclude that subjective empowerment approaches are more likely to be optimistic about the assessment. Additionally, compared to other evaluating methods, the analytic outcome turns out the modified CVM set (CVM1 CVM4) is more preferable.

\section{References}

[1] Liu Yingying, Xu Yonghai, Xiao Xiangning. Analysis of New Method on Power Quality Comprehensive Evaluation for Regional Grid [J]. Proceedings of the CSEE, 2008, 28(22), 130.

[2] Duan Xiangying. Research on the application of maximal tree method based on the fuzzy clustering for power quality evaluation [C]. 2014 China International Conference on Electricity Distribution, Proceedings, 2014, p1284-1287.

[3] Zhou Lin, Li Qiuhua, Liu Huayong, et al. Evaluation of power quality by fuzzy artificial neural network [J].High Voltage Engineering, 2007, 33(9).

[4] Li Nana, He Zhengyou. Power quality comprehensive evaluation combining subjective weight with objective weight [J].Power System Technology, 2009, 33(6).

[5] Zhao Xia, Zhao Chengyong, Jia Xiufang, et al. Fuzzy synthetic evaluation of power quality based on changeable weight[J].Power System Technology, 2005, 29(6): 11-14.

[6] Jiang Hui, Peng Jianchun, Ou Yaping, et al. Power quality unitary quantification and evaluation based on probability and vector algebra [J].Journal of Hunan University: Natural Sciences Edition, 2003, 3(1).

[7] Zhou Lin, Li Qiuhua, Zhang Feng. Application of genetic projection pursuit interpolation model on power quality synthetic evaluation [J].Power System Technology, 2007, 31(7).

[8] Zhang, Gang. The power quality evaluation method based on the matter-element comprehensive evaluation method [J]. Advanced Materials Research, 2014, v1030-1032, 1403-1406.

[9] Wang Dengying. Close value method for optimization of multi-objective decision [J]. System Engineering, 1989, 7(1).

[10]Wang Wenjuan, Wang Ziyan, Chen Tingbin. Study on method of combination weighting in multiple attribute decision making based on association value method[J].Mathematics in Practice and Theory, 2008, 38(13).

[11]Huang Jing, Ma Hongzhong, Ji Hui. Application of intimate data method in synthetical evaluation about power quality [J].Relay, 2008, 36(3).

[12]Chen Yiping. Evaluation of energy saving and emission reduction based on the osculation value method [J].Ecology and Environmental Sciences, 2010, 19(2).

[13]Zhang Tiefeng, Yuan Jinsha, Kong Yinghui. An approach based on AHP/ELECTRE III for decision-aid in power distribution system planning [J].Proceedings of the CSEE, 2006, 26(11), 122.

[14]Men Baohui, Liang Chuan. Attribute recognition model-based variation coefficient weight for evaluating water quality[J].Journal of HarBin Insititute of Technology, 2005, 37(10).

[15]Tao Shun, Xiao Xiangning. Infrastructure of the power quality assessment system of power systems [J].Transaction of China Electrotechnical Society, 2010, 25(4). 
[16]Xu Yonghai, Xiao Xu Yonghai, Xiao Xiangning. Power quality problems in deregulated power systems [J].Power System Technology, 2004, 28(22), 49.

[17]Farghal S A, Kandil M S, Elmitwally A. Quantifying electric power quality via fuzzy modeling and analytic hierarchy processing[J]. IEE Proceedings-generation, Transmission and Distribution, 2002, 149 (1): 44-49.

[18]Li Shiling. A non-liner system modeling method based on projection pursuit and genetic algorithm [J]. Systems Engineering-Theory \& Practice, 2005, 4(4).

[19]Wang, Zongyao. A comprehensive power quality evaluation model based on interval number theory [J]. Power System Protection and Control, 2012, 40(3):41-45. 